\title{
Scaling-Based Analysis and Modelling of Power Losses in Amorphous and Nanocrystalline Alloys
}

\author{
M. NAJGeBAueR* \\ Częstochowa University of Technology, Faculty of Electrical Engineering, \\ al. Armii Krajowej 17, 42-200 Częstochowa, Poland
}

\begin{abstract}
The paper presents the scaling-based approach to analysis and modelling of power losses in Fe-based amorphous and nanocrystalline alloys. Production technology and properties of these alloys are briefly presented. For each sample, a family of measured loss curves are collapsed onto a single one using appropriate estimated scaling parameters. An interpretation of the fractional exponent is discussed. The scaling analysis is used in the modelling of power losses for the considered alloys. The results of power loss modelling and obtained errors are presented.
\end{abstract}

DOI: 10.12693/APhysPolA.131.1225

PACS/topics: 75.50.Bb, 75.60.Ej, 89.75.Da

\section{Introduction}

Power losses in soft magnetic materials are generated by hysteresis phenomenon and eddy currents, flowing in different scales as in the whole material volume, around moving domain walls or micro-currents related to the Barkhausen jumps. Some authors propose considering all types of power losses separately [1-3], while others suggest that these should be considered as total [4].

Modelling of power losses is still an interesting issue both for scientists and researchers. There are many approaches to modelling of power losses. The most popular model has been proposed by Bertotti [1-3]. It is composed of three power law components of frequency and magnetic induction, related to hysteresis, classical and excess losses. This model assumes that power losses are scale-invariant phenomena. On the contrary, some authors propose models composed of two loss components $[5,6]$. A description of power losses in the form of a power law equation is also used [7-9], including technical reports [10].

Referring to multi-scale aspects of power losses, Sokalski et al. have proposed a new approach to the power loss analysis [11, 12], based on the Widom scaling procedure $[13,14]$. The scaling approach allowed collapsing a family of power loss curves onto the single one, given in scaled coordinates by the two-component expression

$$
P_{\text {tot }} / B_{\mathrm{m}}^{\beta}=p_{1}\left(f / B_{\mathrm{m}}^{\alpha}\right)+p_{2}\left(f / B_{\mathrm{m}}^{\alpha}\right)^{2},
$$

where $P_{\text {tot }} / B_{\mathrm{m}}^{\beta}$ are scaled losses, $f / B_{\mathrm{m}}^{\alpha}$ is scaled frequency, $\alpha, \beta$, and $p_{1,2}$ are scaling parameters. However the expression (1) has some limitations, because exponents of the scaled frequency can be only integers. Recently, a modified approach to scaling analysis of power losses has been proposed, in which exponents of scaled frequency were fractional numbers. The fractional scal-

\footnotetext{
*e-mail: najgebauer@el.pcz.czest.pl
}

ing provided a more general expression, describing scaled losses

$$
\begin{aligned}
& P_{\text {tot }} / B_{\mathrm{m}}^{\beta}=p_{1}\left(f / B_{\mathrm{m}}^{\alpha}\right)^{x}+p_{2}\left(f / B_{\mathrm{m}}^{\alpha}\right)^{2 x} \\
& \quad+p_{3}\left(f / B_{\mathrm{m}}^{\alpha}\right)^{3 x} \ldots,
\end{aligned}
$$

where $x$ is a fractional exponent. It was further found for grain-oriented electrical steels that the use of expression (2) in the reduced form

$$
P_{\text {tot }} / B_{\mathrm{m}}^{\beta}=p\left(f / B_{\mathrm{m}}^{\alpha}\right)^{x},
$$

gave comparable results of power loss scaling [15]. The fractional scaling has been also applied in the analysis of power losses for La-containing magnetocaloric alloys [16], yielding promising results.

In the present study, the expression (3) is examined in scaling analysis and modelling of power losses for Febased alloys with amorphous and nanocrystalline internal structures.

\section{Materials and measurements}

Amorphous alloys are produced by solidification of a molten metallic alloy on the rotating drum surface, which causes an alloy transformation from a liquid state into a solid one. This process is so rapid that a crystallization structure cannot be created inside the material, which results in a metastable amorphous (glass) state. Magnetic properties of amorphous alloys depend on their chemical composition, but - in general - these alloys have lower values of power losses and magnetic induction compared to electrical steels. Nanocrystalline alloys are obtained by partial crystallization of amorphous ribbons, during which crystalline grains are created inside an amorphous matrix. This process changes the magnetic properties of the alloy in addition to making it more thermally stable. Amorphous alloys are generally used in distribution transformers and electric motors, whereas nanocrystalline alloys find application in high frequency electric and electronic devices [17-21].

In the study, samples of commercially produced Febased amorphous and nanocrystalline alloys are ana- 
lyzed. The amorphous sample was made of $\mathrm{Fe}_{78} \mathrm{Si}_{13} \mathrm{~B}_{9}$ alloy as quenched, whereas the nanocrystalline one was made of $\mathrm{Fe}_{73.5} \mathrm{Cu}_{1} \mathrm{Nb}_{3} \mathrm{Si}_{13.5} \mathrm{~B}_{9}$ alloy after thermal treatment leading to a Finemet-type structure. Both samples have cylindrical shapes with dimensions (outer radius, inner radius and height) $27.2 \times 13.5 \times 10.5 \mathrm{~mm}^{3}$ and $12.5 \times 8.0 \times 10.0 \mathrm{~mm}^{3}$ for amorphous and nanocrystalline ones, respectively.

Power loss measurements for these samples were carried out using the computer-aided measurement system REMACOMP C-200, according to the IEC international standard. The measurement ranges were: $50 \mathrm{~Hz}$ to $400 \mathrm{~Hz}$ for frequency and $0.1 \mathrm{~T}$ to $1.2 \mathrm{~T}$ with increment $0.1 \mathrm{~T}$ for magnetic induction. The measurements were carried out at a fixed value of magnetic induction and for varying values of excitation frequency, providing a family of power loss curves.

\section{Results and discussion}

The parameters $p, x, \alpha$ and $\beta$ were estimated from a family of measured loss curves for each tested sample, using the least-squares optimization based on the generalized reduced gradient method. A correct estimation of parameters should allow collapsing all loss curves onto a single one, given in scaled coordinates. In this case, parameter values could be considered as universal in the whole measured range. An idea of power loss scaling for the Fe-based amorphous sample is presented in Fig. 1. A similar result of loss scaling was obtained for the nanocrystalline sample, which is presented in Fig. 2.

The quality of proposed loss scaling was evaluated by calculating the coefficient of determination $R^{2}$. Values of the estimated parameters and the coefficient $R^{2}$ for the tested samples are given in Table I. The values of coefficient $R^{2}$ are very close to 1 , which means that scaled measurements are fitted well to a data collapse curve, described by the expression (3). Thereby, it confirms that the power loss scaling was carried out correctly. The fractional exponent $x$ is equal to 1.36 and 1.31 for the amorphous/nanocrystalline samples, whereas for samples with crystalline structures it is about 1.40 and 1.63, respectively for non-oriented and grain-oriented steels [15]. So, its value depends on a type of internal structure and geometry of the sample. For this reason, the fractional exponent $x$ may be considered as a material parameter, correlated with an internal structure of the sample as well as predominant mechanisms of energy dissipation, determined by the sample geometry.

TABLE I

Parameters and coefficient $R^{2}$ of power loss scaling.

\begin{tabular}{c|c|c|c|c|c}
\hline \hline Sample & $p$ & $\alpha$ & $\beta$ & $x$ & $R^{2}$ \\
\hline amorphous & $0.56 \times 10^{-3}$ & -2.36 & -1.64 & 1.36 & 0.9985 \\
\hline nanocrystalline & $0.38 \times 10^{-3}$ & -2.38 & -0.89 & 1.31 & 0.9974
\end{tabular}

In the proposed scaling-based modelling of power losses the expression (3) was used. The expression (3) was converted to the form that describes power losses directly
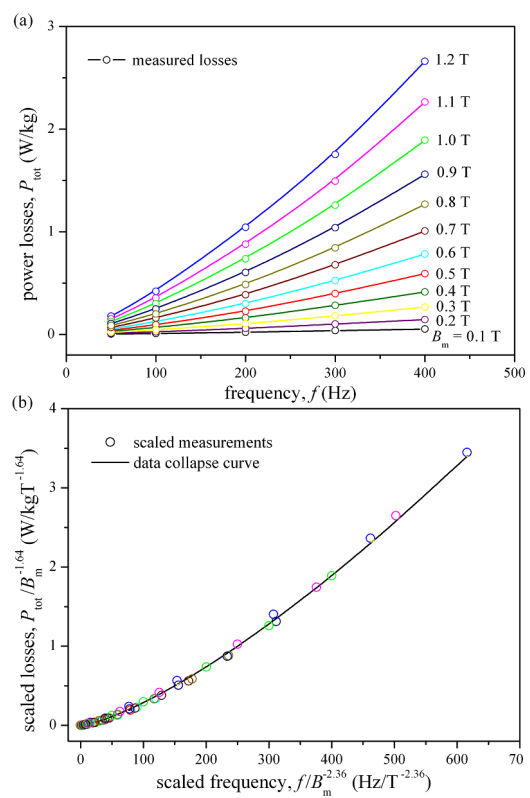

Fig. 1. An idea of power loss scaling for the Fe-based amorphous sample: (a) a family of measured loss curves, (b) result of loss scaling (a data collapse curve).

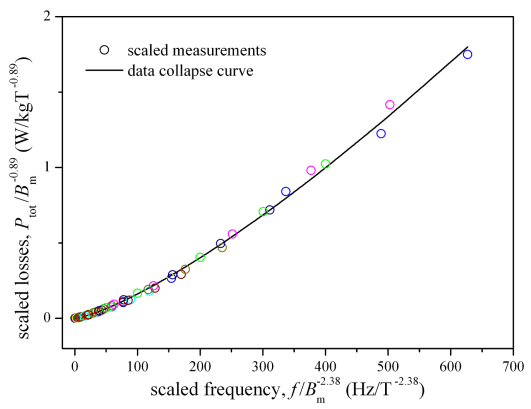

Fig. 2. Power loss scaling for the Fe-based nanocrystalline sample.

$P_{\mathrm{tot}}=p f^{x} B_{\mathrm{m}}^{y}$,

where $y=\beta-\alpha x$. The exponents $x$ and $y$ were calculated for each sample, using scaling parameters presented in Table I, which gave the following formulae for power losses:

- for Fe-based amorphous alloy

$P_{\text {tot }}=p f^{1.36} B_{\mathrm{m}}^{1.57}$,

- and for Fe-based nanocrystalline alloy

$P_{\text {tot }}=p f^{1.35} B_{\mathrm{m}}^{2.24}$.

Power losses were modelled according to the formulae (5), (6) for frequency varying from $50 \mathrm{~Hz}$ to $400 \mathrm{~Hz}$ and arbitrary chosen values of maximum induction equal to $B_{\mathrm{m}}=0.5,1.0$, and $1.2 \mathrm{~T}$. Results of the scaling-based modelling of power losses for the considered cases are presented in Figs. 3 and 4.

The uncertainties of power loss modelling were determined by calculating the percentage errors in each modelled point. Additionally, mean percentage errors (MPE) of modelled loss curves were calculated. The values of 


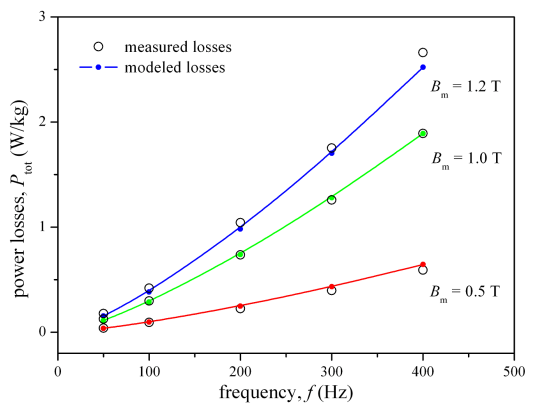

Fig. 3. Scaling-based modelling of power losses for the amorphous sample: solid line - modelled losses, circles - measurements.

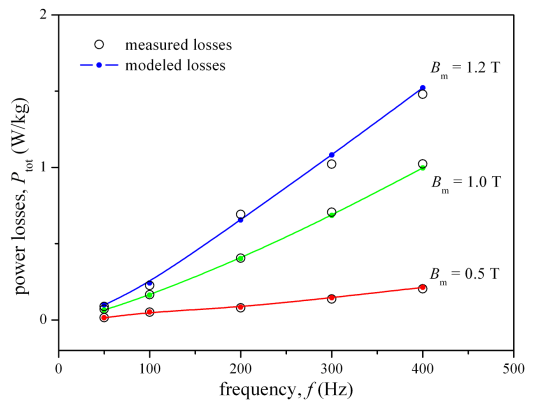

Fig. 4. As in Fig. 3 but for the nanocrystalline sample.

minimum/maximum errors and MPEs are presented in Table II. Maximum errors of the scaling-based loss modelling are about $10-13 \%$, whereas minimum errors do not exceed $3.3 \%$ and for several points are even lower than $1 \%$. The MPE values vary from $3.2 \%$ up to $7.8 \%$, depending on the sample and maximum induction. The obtained values of modelling errors are relatively small. Thereby, the results of power loss modelling may be considered as satisfactory.

\section{TABLE II}

Percentage errors of scaling-based modelling of power losses.

\begin{tabular}{l|c|c|c}
\hline \hline Sample & $\delta_{\min }[\%]$ & $\delta_{\max }[\%]$ & $\delta_{M P E}[\%]$ \\
\hline amorphous, at 0.5 T & 3.3 & 10.5 & 7.8 \\
\hline amorphous, at 1.0 T & 0.4 & 11.4 & 3.4 \\
\hline amorphous, at 1.2 T & 2.8 & 12.6 & 7.1 \\
\hline nanocrystalline, at 0.5 T & 1.2 & 7.0 & 5.0 \\
\hline nanocrystalline, at 1.0 T & 0.8 & 6.9 & 3.2 \\
\hline nanocrystalline, at 1.2 T & 2.8 & 10.2 & 6.4
\end{tabular}

\section{Conclusions}

In the present study, the scaling-based approach to power loss analysis and modelling was examined for Febased amorphous and nanocrystalline alloys. Measured curves of power losses were collapsed onto a single curve, which might be considered as verification of scaling behaviour of magnetic losses in amorphous and nanocrystalline alloys. An interpretation of the fractional param- eter $x$ was proposed as well as a possible correlation between this parameter and an internal structure of the sample was pointed out.

The fractional scaling was used in modelling of power losses for considered alloys. Results of the scaling-based modelling of power losses were satisfactory due to relatively low discrepancies between measured and modelled loss curves. The maximum modelling error did not exceed $12.6 \%$ whereas minimum errors were lower than $3.3 \%$. These results prove that the fractional scaling procedure provides satisfactory results of power loss modelling for a wide class of soft magnetic materials, having different internal structures.

Finally, the fractional scaling is an interesting contribution to the issue of power loss modelling for soft magnetic materials, because it does not require any information concerning sample geometry or material constants, which makes the modelling easier.

\section{References}

[1] G. Bertotti, J. Magn. Magn. Mater. 41, 253 (1984).

[2] G. Bertotti, IEEE Trans. Magn. 24, 621 (1988).

[3] G. Bertotti, Hysteresis in Magnetism, Academic Press, San Diego 1998.

[4] B.D. Cullity, C.D. Graham, Introduction to Magnetic Materials, 2nd ed., Wiley-IEEE Press, Hoboken 2009.

[5] J. Barranger, Hysteresis and Eddy-Current Losses of a Transformer Lamination Viewed as an Application of the Poynting Theorem, NASA Technical Note TN D-3114, Washington D.C. 1965.

[6] K. Chwastek, Philos. Mag. Lett. 90, 809 (2010).

[7] S. Mulder, Power Conv. Intellig. Motion 21, 22 (1995).

[8] K. Venkatachalam, C.R. Sullivan, T. Abdallah, H. Tacca, in: Proc. IEEE Workshop on Computers in Power Electronics, Puerto Rico, 2002, p. 36.

[9] K. Sokalski, J. Szczygłowski, W. Wilczyński, Int. J. Condens. Matter 12, 1 (2014).

[10] Finemet. Square loop cores, Technical Bulletin, Metglas, Inc..

[11] K. Sokalski, J. Szczyglowski, M. Najgebauer, W. Wilczynski, COMPEL 26, 640 (2007).

[12] M. Najgebauer, Prz. Elektrotechn. 84, 213 (2008).

[13] B. Widom, J. Chem. Phys. 43, 3898 (1965).

[14] H.E. Stanley, Rev. Mod. Phys. 71, S358 (1999).

[15] M. Najgebauer, Acta Phys. Pol. A 128, 107 (2015).

[16] R. Gozdur, M. Najgebauer, J. Electr. Eng. 66, 37 (2015).

[17] G. Herzer, Scr. Metall. Mater. 33, 1741 (1995).

[18] M.E. McHenry, M.A. Williard, D.E. Laughlin, Prog. Mater. Sci. 44, 291 (1999).

[19] R. Hasegawa, J. Magn. Magn. Mater. 215-216, 240 (2000).

[20] R. Hasegawa, J. Magn. Magn. Mater. 304, 187 (2006).

[21] M. Najgebauer, J. Szczygłowski, Prz. Elektrotechn. 84, 136 (2008). 